\title{
Risikomanagement in Beschaffung und Distribution - Entwicklung eines tech- nologieorientierten Maßnahmenrasters zur Schadenverhütung im Transport
}

\author{
Alexander C. H. Skorna, Philipp Hirsbrunner und Christoph Bode
}

\section{Abstract}

Warentransporte als Bindeglied zwischen den global verteilten Beschaffungs- und Absatzmärkten sind einer Vielzahl von Risiken und Gefahren ausgesetzt. Durch die zunehmende Einbindung aufstrebender Schwellenländer in die globale Warenwirtschaft erhöht sich die dynamische und strukturelle Komplexität der Transportnetzwerke zusätzlich. Wirtschaftlich können gehäuft auftretende Transportschäden bei Unternehmen zu Betriebsunterbrechungen oder einer eingeschränkten Lieferfähigkeit führen, die die Kosten eines einzelnen Transportschadens meist weit übersteigen. Im Kontext des Risikomanagements von Beschaffungs- und Distributionsprozessen wird in diesem Beitrag ein Maßnahmenraster zur Schadenverhütung im Transport entwickelt. Die Ergebnisse werden in Form eines Technologieportfolios visualisiert, welches die wesentlichen Hauptursachen für Transportschäden adressiert. Das Schadenreduktionspotenzial der Maßnahmen wird im Anschluss anhand von Expertenbefragungen validiert. Der Beitrag schließt mit konkreten Handlungsempfehlungen für die Praxis.

\section{$1 \quad$ Problemstellung}

Das aktuelle Umfeld von Transport- und Logistikprozessen ist durch eine zunehmende Globalisierung, steigenden Wettbewerbsdruck sowie gestiegene Anforderungen der Endkunden hinsichtlich Preis, Qualität und Verfügbarkeit von Waren gekennzeichnet. Unternehmen konkurrieren heute eher beim Zugang zu neuen Beschaffungsund Absatzmärkten als bei der eigentlichen Produktherstellung. Unternehmen nutzen vor allem die Lohnkostenunterschiede zwischen den Ländern und versuchen, neue Absatzmärkte für sich zu erschließen. Lieferanten, Produzenten und Distributoren arbeiten daher vielfach eng zusammen und bilden Wertschöpfungsnetzwerke. Logis- 
tikdienstleister integrieren ihrerseits Liefer- und Transportketten als Bindeglieder zwischen den einzelnen Unternehmen und unterstützen die Unternehmen so zusätzlich, nachhaltige Wettbewerbsvorteile zu erzielen (Christopher/Towill 2002). Gleichzeitig steigen die Transportmengen und transportierten Warenwerte, da Unternehmen Fertigungsprozesse ausgelagert haben und so hochwertige Zwischen- und Halbfertigprodukte zukaufen, bevor sie die Endprodukte in die unterschiedlichsten Absatzmärkte senden. Der Anstieg des grenzüberschreitenden Transportvolumens und die Einbindung mehrerer Transportakteure führen somit zu einer steigenden Komplexität der Transportprozesse (Pfohl 2002).

Bei der Optimierung der Logistiknetzwerke unter diesen Rahmenbedingungen einer multipolaren Welt zeichnet sich ein Spannungsverhältnis aus Transportzeit, Qualität und Kosten ab. Die größeren Transportdistanzen, eine Einbindung von vergleichsweise unterentwickelten Transportnetzwerken und unerfahrene Logistikmitarbeiter machen die Logistik insbesondere in Schwellenländern zu einem schwer beherrschbaren Prozess (Skorna/Hinz 2011, S. 27). Die Folge dieser Entwicklung ist letztlich ein Anstieg der Verwundbarkeit von globalen Wertschöpfungsnetzwerken. Neben der Globalisierung der Wertschöpfungsnetzwerke hat Jüttner (2005) den Abbau von Beständen und die zunehmende Tendenz des Outsourcings sowie die konsequente Anwendung von Lean-Management-Methoden in Supply Chains als wesentliche Treiber der Verwundbarkeit identifiziert (Jüttner 2005, S. 134). In diesem Zusammenhang ergänzt Peck (2005), dass eine steigende Abhängigkeit von Lieferanten und eine hohe Variabilität der Nachfrage bei produzierenden Unternehmen zu einer größeren Anfälligkeit von Betriebsunterbrechungen führen.

Vor dem Hintergrund dieser skizzierten Problemstellung ist das Ziel dieses Beitrags die Entwicklung eines technologieorientierten Maßnahmenrasters zur Schadenverhütung in Transportprozessen. Im Zentrum stehen dabei Technologien aus dem Bereich des Internets, die einerseits die Transparenz in der Transportkette erhöhen und andererseits Transportschäden frühzeitig anzeigen. Zusammen mit organisatorischen Maßnahmen können diese Technologien das Ausmaß eines bereits aufgetretenen Transportschadens mildern oder im Idealfall einen Schaden ganz verhindern. Hierzu gliedert sich dieser Beitrag in die folgenden drei Abschnitte: Kapitel 2 dient einer Rekapitulation von relevanten Grundlagen und Definitionen sowie verschiedenster organisatorischer Maßnahmen aus dem Bereich des operativen Risikomanagements von Transportprozessen. Zusätzlich werden die wesentlichen Hauptursachen von Transportschäden sowohl aus der Literatur als auch anhand einer empirischen Analyse von 19.180 Schadenfällen eines der größten Europäischen Transportversicherungsunternehmen hergeleitet. Das technologieorientierte Maßnahmenraster wird in Kapitel 3 jeweils separat für die Schadenursachen (1) Erschütterungen, (2) Neigung und Kippen, (3) Temperatur- und (4) Nässeeinwirkung sowie (5) Diebstahl und Verlust auf der Basis des Technologieportfolios nach Pfeiffer (1991) explorativ entwickelt. Die Vorarbeiten der Autoren wurden im Rahmen einer qualitativen Expertenbefragung (Meuser/Nagel 2005) überprüft und zu dem in diesem Beitrag präsentierten Maßnahmen- 
raster weiterentwickelt. Gegenstand des anknüpfenden Kapitels 4 ist die Aufbereitung der Ergebnisse zu konkreten Handlungsempfehlungen für die Praxis.

\section{Risikomanagement im Transport}

\subsection{Grundlagen und Definitionen}

Das Risikomanagement in Lieferketten fasst sämtliche Strategien, Maßnahmen, Prozesse und Technologien zusammen, um Risiken in einer Lieferkette zu reduzieren (Christopher/Peck 2004; Jüttner 2005). Dabei handelt es sich in Anlehnung an Kummer/Sudy (2007) und Rogler (2002) insbesondere um:

die Vermeidung von Risiken durch die aktive Eliminierung von möglichen Schadenursachen;

die Überwälzung der Risiken auf externe Parteien als „Outsourcing“ an z. B. Lieferanten, Kunden, Transport- und Logistikdienstleister oder in Form einer Übertragung durch Verträge z. B. mit Transportversicherungen;

die bewusste Selbsttragung der Risiken und das eigenverantwortliche Risikomanagement z. B. durch Interventionspläne, die das Schadenausmaß begrenzen.

Schadenverhütungsmaßnahmen im Kontext von Transport und Logistik beziehen sich auf Prozesse und Maßnahmen, die die Eintrittswahrscheinlichkeit eines Schadens reduzieren oder schädliche Auswirkungen auf einen spezifischen Akteur verringern (Zsidisin und Ellram 2003). Für den Begriff des Risikos gibt es in der Literatur eine Vielzahl unterschiedlicher Definitionen (Kummer/Sudy 2007, S. 256). Im Zusammenhang eines Lieferkettenmanagements wird der Risikobegriff anhand zwei sich ergänzender Begriffsverständnisse definiert. Die ursachenbezogene Risikodefinition basiert auf unvollkommenen Informationen bei unternehmerischen Entscheidungen (Kajüter 2007, S. 14; Steven/Pollmeier 2007, S. 274). Daher kann eine ideale Zielerreichung durch ein Auftreten zukünftiger, meist unerwarteter Entwicklungen beeinträchtigt werden. Die wirkungsbezogene Risikodefinition stellt eine mögliche negative Abweichung1 ins Zentrum. So werden Risiken unter Einbezug beider Sichtweisen als zukünftige Entwicklungen und Ereignisse definiert, die aufgrund unvollkommener Informationen die Nichterreichung von Zielen auf Unternehmensebene oder bezogen auf die gesamte Lieferkette bewirken können (Kersten/Blecker 2006, S. 5). Bezogen auf Liefer- und Transportrisiken wird in Anlehnung an Zsidisin (2001) und March/Shapira (1987) eine gefahrenorientierte Interpretation des Risikos verstanden. Ein Risiko ergibt

$1 \quad$ Allgemein kann diese Abweichung auch positiv sein (Kajüter 2007, S. 15). 
sich demnach aus der Eintrittswahrscheinlichkeit (eines spezifischen Schadens) multipliziert mit dem Ausmaß (einer negativen Auswirkung auf das Unternehmen bzw. die Lieferkette).

Das Risikomanagement bzw. die Ausgestaltung von Maßnahmen zur Schadenverhütung im Transport unterliegt durch den unternehmensübergreifenden Charakter einigen Besonderheiten. Diese liegen insbesondere im Bereich der höheren Komplexität und der unternehmensübergreifenden Organisation der Prozesse (Wagner/Bode 2007, S. 67). In Anlehnung an Kajüter (2007) werden im Folgenden weitere wesentliche Besonderheiten aufgeführt:

Der Handlungsrahmen des Risikomanagements erweitert sich von der Unternehmensebene zur Ebene ganzer Lieferketten bzw. Transportnetzwerke.

Es bestehen Informationsasymmetrien zwischen den einzelnen Unternehmen.

Die Summe aller Risiken der beteiligten Unternehmen unterscheidet sich von den Risiken des Transportnetzwerks.

Es bestehen Unterschiede betreffend Risikobereitschaft und Risikotragfähigkeit unter den Unternehmen in der Lieferkette.

Die Bereitschaft und Möglichkeit einer Unternehmung, sich an Standards einer einzelnen Lieferkette zu orientieren, sind häufig aufgrund der Zugehörigkeit zu mehreren Lieferketten begrenzt.

Internationale Lieferketten und Transporte bergen die Schwierigkeit regionaler und kultureller Faktoren, die gesondert zu berücksichtigen sind.

Jüttner et al. (2003) argumentieren, dass der Begriff Risiko in Risikoquellen und Risikokonsequenzen unterteilt werden sollte. Die Risikoquellen und Konsequenzen werden in Form von Schadenursachen bzw. Schadenarten in Kapitel 2.2 noch genauer identifiziert. Das Transportrisiko kann allerdings je nach vereinbarter Lieferklausel („Incoterms“2) gänzlich unterschiedliche Partner in der globalen Lieferkette betreffen (Rogler 2002). Obwohl sich Unternehmen zunehmend mit Schadenverhütungsmaßnahmen beschäftigen, sind nur wenige konkrete und situativ angepasste Handlungsempfehlungen zur Reduzierung von Transportschäden verfügbar (Autry/Bobbit 2008).

Die inzwischen weit verbreitete Nutzung von Informations- und Kommunikationstechnologien hat zu einer Entwicklung von schnelleren, verlässlicheren und präzise aufeinander abgestimmten Logistik- und Transportprozessen geführt. Transportdienstleistungen sind damit zu informationsintensiven Abläufen geworden. Die Herausforderung bei der Entwicklung eines technologieorientierten Maßnahmenrasters zur Schadenverhütung besteht nun darin, diese Ansätze meist aus dem Bereich der Sendungsverfolgung zu nutzen. Das Risikomanagement der unterschiedlichen Partner

2 Abkürzung für International Comercial Terms (dt.: Internationale Handelsklauseln). 
eines Transportnetzwerks ist bisher stark auf das Schadenmanagement und den Risikotransfer fokussiert (vgl. Abbildung 1).

Abbildung 1: Risikomanagementprozesse im Transport (Skorna/Bode/Wagner 2009, S. 209)

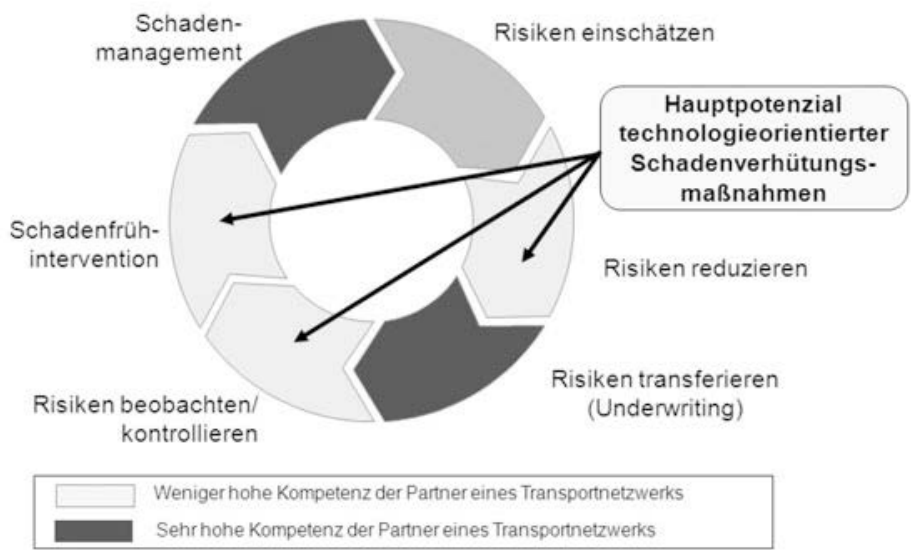

Die Handhabung von Risiken in einem Transportnetzwerk erfordert zunächst die Identifikation von Risikoereignissen und Schwachstellen. Risiko-Assessments basieren oft auf standardisierten Verfahren und bleiben vage in ihrer Belastbarkeit bzw. Aussagefähigkeit (vgl. Abbildung 1, dunkle Prozessschritte). Operative Risikomanagementprinzipien erweitern die klassische Prozesskette hinsichtlich der Beratung zur Schadenverhütung, Unterstützung bei der Risikokontrolle und Kooperation im Bereich technologieorientierter Schadenfrühintervention (vgl. Abbildung 1, helle Prozessschritte). Durch die Einführung von Schadenverhütungsmaßnahmen im Transport werden insbesondere Risikofaktoren minimiert oder Schutzfaktoren verbessert. Ihr Ziel ist eine Beeinflussung der Einflussfaktoren, damit sich die Wahrscheinlichkeit für das Auftreten eines Schadenereignisses reduziert bzw. die mittlere Schadenhöhe des Ereignisses möglichst gering bleibt (Skorna/Bode/Fleisch 2010, S. 15). Die Abbildung 1 beschreibt diesen Zusammenhang anhand eines generischen Risikomanagementprozesses, wie er sich für die Beteiligten eines Transports allgemein darstellt. Das Hauptpotenzial technologieorientierter Schadenverhütungsmaßnahmen liegt somit in einer Erhöhung der Sehschärfe auf die Transportprozesse. Dadurch werden Informationen über Transportrisiken gewonnen, die zur schrittweisen Risikoreduktion und einer kontinuierlichen Risikobeobachtung verwendet werden können. Über einen verbreiteten Technologieeinsatz nehmen die zur Risikobeurteilung verfügbaren Daten durch Transportüberwachung weiter zu.

Nachfolgend werden Transportrisiken und Schadenursachen anhand einer empirischen Analyse von Schadendaten einer Transportversicherung identifiziert. Dieses 
Vorgehen schafft ein notwendiges Vorverständnis über aktuelle Problemstellungen in der Transportlogistik.

\subsection{Identifikation von Transportrisiken und Schadenursachen}

Transportrisiken umfassen alle Einzelrisiken, die im Zusammenhang mit dem physischen Transport der Güter stehen (Eberle 2005, S. 74). Die Identifikation sowie Bewertung von Transportrisiken und ihren Auswirkungen in einer Lieferkette ist eine komplexe und teils komplizierte Aufgabe (Pfohl 2010). Typische Risikoquellen im Transport sind nach Peck (2005) und Mason-Jones/Towill (1998) Verluste beispielsweise durch Diebstahl und Raub, Beschädigungen durch Erschütterung und Kippen bzw. das Verderben von Waren infolge Temperatur- und Nässeeinfluss sowie Lieferverzögerungen.

In der Literatur existieren bereits Ansätze, wie mit neuen Methoden aus der Versicherungsindustrie die bestehenden Bezugsrahmen zur Risikoanalyse erweitert werden können (Knemeyer et al. 2009, S. 142). Über die Einbindung der Transportversicherer als kompetente Schadenmanager können Transportprozesse hinsichtlich ihrer potenziellen Gefahren optimiert werden (Skorna et al. 2010, S. 14). In diesem Zusammenhang weisen Norrman und Jansson (2004) darauf hin, dass Transportversicherungsgesellschaften die treibende Kraft für Verbesserungen im Sinne des Risikomanagements sein könnten, da sie die Anfälligkeit von modernen, globalen Lieferketten zunehmend verstehen. Um noch genauere Hinweise betreffend Frequenz und Höhe der Risikoquellen bzw. Schadenursachen zu ermitteln, wurden insgesamt 19.180 Schäden aus dem Schadensystem einer Schweizer Transportversicherung über einen Zeitraum von zehn Jahren analysiert (Januar 2001 bis Dezember 2010). Aufgrund der Zeitdifferenz zwischen Schadeneintritt und Schadenmeldung sind die Daten erst im Juli 2011 aus dem Schadensystem der Versicherung zusammengestellt worden. So ist eine vollständige Berücksichtigung des Jahres 2010 gewährleistet. Die Transporte sind dabei weltweit verteilt, regionale Schwerpunkte bilden Europa, der Mittlere Osten, Nordafrika sowie Nordamerika und Asien.

Die Analyse erfolgt aus zwei unterschiedlichen bzw. gegensätzlichen Richtungen, die die im vorherigen Kapitel beschriebenen Zusammenhänge aus Eintrittswahrscheinlichkeit (Frequenz) und Ausmaß (effektive Höhe) eines Schadens widerspiegelt. (1) Aus Sicht der Versicherungsgesellschaft ist zunächst der effektive Schadenaufwand pro Schadenereignis von Bedeutung. Für die Entwicklung eines Konzepts zur Schadenverhütung von Transportschäden aus der Versicherungsperspektive ist es relevant, mit zielgerichteten Maßnahmen langfristig die Schadenzahlungen zu minimieren. Aus der Sicht des Versicherungsnehmers spielt der effektive Schadenaufwand bei gegebener Deckung meist eine untergeordnete Rolle. (2) Versicherungsnehmer sind dagegen daran interessiert, häufig eintretende Frequenzschäden während des Transports zu vermei- 
den und dadurch ihre Liefer- und Produktqualität den Endkunden gegenüber sicherzustellen.

Die Abbildung 2 stellt den Verlauf von jährlichen Schadenaufwendungen und der Schadenfrequenz über den Beobachtungszeitraum dar. Zwischen den Jahren 2002 und 2009 ist die Schadenfrequenz relativ konstant. Im Jahr 2010 ergibt sich eine leicht steigende Tendenz bei gleichzeitig sinkenden Schadenaufwendungen. Die Schadenbelastung ist insgesamt deutlich volatiler, da das jährliche Schadenvolumen durch ein Auftreten von seltenen Großschadenereignissen geprägt wird. So lässt sich der Ausbruch der Schadenbelastung im Jahre 2005 durch einen einzelnen Schadenfall in der Höhe von 5,2 Mio. SFr. erklären. Es handelte sich hier um den Verlust hochwertiger Pharmazeutika bei einem Lkw-Transport innerhalb der Vereinigten Staaten (USA). In den übrigen Zeitperioden liegen die maximalen Schadenaufwendungen für ein einzelnes Schadenereignis nur etwa halb so hoch.

Abbildung 2: Zeitlicher Verlauf der jährlichen Schadenaufwendungen und Schadenanzahl

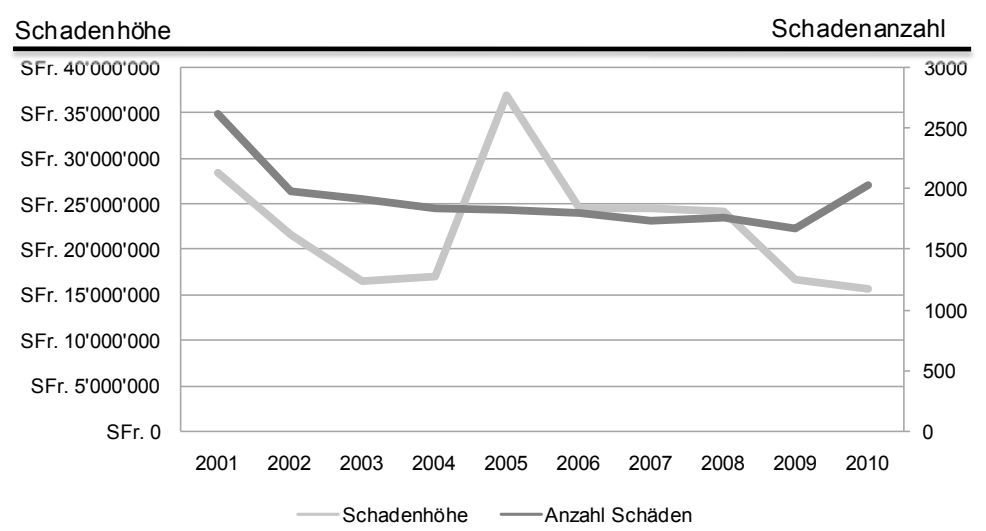

Bei den Schadenarten dominieren die Beschädigung von Waren mit einem Anteil von 63 \% der Anzahl Schäden bzw. 49 \% der Schadenaufwendungen sowie der Verlust von Waren mit einem Anteil von 27 \% Anzahl Schäden bzw. 42 \% der Schadenaufwendungen. Damit stellt sich die Warenbeschädigung als typischer Frequenzschaden dar, der Warenverlust dagegen verursacht relativ gesehen höhere Schadenaufwendungen. ${ }^{3}$

Tabelle 1 beschreibt die Hauptschadenursachen und die von Schäden betroffenen Verkehrsträger jeweils nach ihrem Anteil an der Schadenanzahl und den Schadenaufwen-

3 Eine ausführliche Analyse der Schadenursachen findet sich auch bei Skorna et al (2009), S. 201. 
dungen. Auch hier lassen sich die Schadenursachen und Verkehrsträger als eher anfällig für Frequenzschäden (Kennzeichnung * in Tabelle 1) bzw. hohe Schadenbelastungen aufteilen. Einschränkend wird an dieser Stelle erwähnt, dass die Angaben nur absolute Schadeneinträge in der Schadenhistorie darstellen und die Exposition z. B. der Verkehrsträger weitgehend unberücksichtigt bleibt. In der Seefracht sind bei einem Schadenfall üblicherweise deutlich mehr Waren betroffen als z. B. in der Luftfracht mit wesentlich geringeren Durchschnittsgewichten pro Sendung. Außerdem wird der Vorbzw. Nachlauf von See- und Luftfrachtsendungen üblicherweise auch über Straßentransporte zugestellt. Die Zuordnung der Schäden bezieht sich in der Datenbank jeweils nur auf den Hauptverkehrsträger. Der Schaden kann aber je nach Schadenort bzw. Zeitpunkt von einem anderen Verkehrsträger verursacht worden sein. Die Schadenursache Feuer/Explosion bezieht sich nahezu ausschließlich auf die Lagerung bzw. systembedingte Zwischenlagerung der Güter während eines Transports. Hier bewirkt die vergleichsweise große Akkumulation von Warenwerten die zu beobachtenden hohen Anteile an dem gesamten Schadenaufwand. Die „Havarie Grosse“ ist eine Bezeichnung aus der Seefracht. Sie wird von den beteiligten Versicherungen festgestellt, wenn aufgrund der außergewöhnlichen Umstände bewusst Schäden bzw. Kosten in Kauf genommen werden, um Schiff und Ladung aus einer gemeinsamen Gefahr zu retten (Wagner 2010, S. 294).

Tabelle 1: $\quad$ Hauptschadenursachen (a) sowie von Schäden betroffene Verkehrsträger (b)

\begin{tabular}{|l|c|c|l|c|c|}
\hline \multicolumn{3}{|c|}{ A: Schadenursache } & \multicolumn{3}{c|}{ B: Verkehrsträger } \\
\hline & Anteil Anzahl & Anteil Höhe & & Anteil Anzahl & Anteil Höhe \\
\hline Handhabung * & $58 \%$ & $29 \%$ & Lkw ${ }^{*}$ & $45 \%$ & $32 \%$ \\
\hline Diebstahl & $22 \%$ & $23 \%$ & Seeschiff & $25 \%$ & $33 \%$ \\
\hline Sturz ${ }^{*}$ & $5 \%$ & $3 \%$ & Flugzeug & $13 \%$ & $17 \%$ \\
\hline Wasser & $3 \%$ & $4 \%$ & Post/Express * & $7 \%$ & $3 \%$ \\
\hline Ladungsmangel & $2 \%$ & $2 \%$ & Ausstellungen * & $4 \%$ & $2 \%$ \\
\hline Unfall & $2 \%$ & $5 \%$ & Lagerung & $2 \%$ & $8 \%$ \\
\hline Feuchtigkeit & $1 \%$ & $5 \%$ & Kran/Stapler & $1 \%$ & $1 \%$ \\
\hline Raubüberfall & $1 \%$ & $11 \%$ & Pkw * & $1 \%$ & $>1 \%$ \\
\hline Havarie Grosse & $1 \%$ & $1 \%$ & Bahn & $1 \%$ & $1 \%$ \\
\hline Feuer/Explosion & $>1 \%$ & $5 \%$ & SicherheitsFzg. & $>1 \%$ & $3 \%$ \\
\hline
\end{tabular}

Für die Entwicklung eines technologieorientierten Maßnahmenrasters zur Schadenverhütung bedeutet dies, dass die Schadenursachen überwiegend den folgenden fünf Risikoquellen zuzuordnen sind (Skorna/Hinz 2011, S. 24; Skorna et al. 2010, S. 16):

Erschütterungen, verursacht durch mechanisch-dynamische Beanspruchungen und abrupte Beschleunigungen von Waren. Daneben können auch statische Belastungen aus dem Stapeldruck der Waren Erschütterungen verursachen. Bezogen auf obige Schadenursachen können Erschütterungen aus roher Handhabung, Sturz, Ladungsmangel und Unfall resultieren. 
Schäden durch Neigung und Kippen der Waren treten üblicherweise durch Umfallen infolge einer seitlichen Belastung der Packstücke auf. Vor allem beim Transport von Flüssigkeiten ist die Neigung kritisch, da sich beim Kippen der Ware gleichzeitig der Schwerpunkt verschiebt. Der Transport von Großmaschinen, deren Schwerpunkt nicht mittig liegt, ist generell anfälliger für ein Kippen während der Auf-/ Umladung.

Der Risikofaktor Temperatur betrifft vorrangig Lebensmittel, Spezialchemikalien und Pharmazeutika. Die teilweise sehr engen Temperaturkorridore, in denen die Waren transportiert werden, lassen die Logistik und den Transport zu einer komplexen Aufgabe werden. Für temperatursensitive Waren ist eine durchgehende Überwachung der Temperatur und Kühlung während des Transports erforderlich, um Haltbarkeit und Qualität zu gewährleisten. Stark schwankende, zu hohe und zu niedrige Transporttemperaturen führen zu Qualitätsminderungen bzw. Beschädigungen. Je genauer die Temperaturen während des Transports den Anforderungen der Ware angepasst werden können, umso besser kann deren Qualität und Warenwert gehalten werden.

Eine Nässeeinwirkung kann bei Warentransporten entweder direkt z. B. durch Starkregen und Seewasser oder indirekt z. B. durch die Bildung von Schweißwasser bzw. Kondensation erfolgen. Zusätzlich kann ein transportwegebedingter Anstieg der Feuchte im Laderaum z. B. im Tropengebiet zu einer übermäßigen Schimmelbildung oder zu Korrosion führen. Oft wird dabei das Klima des Frachtraums derart verändert, dass die komplette Ladung beeinträchtigt wird und so vergleichsweise hohe Schadenaufwendungen verursacht werden.

Der Verlust von Waren verursacht durch Diebstahl, Abhandenkommen oder administrative Fehler ist eine der signifikantesten Bedrohungen für die Profitabilität von Unternehmen. Addiert man Diebstahl und Raub, so sind diese beiden Ursachen mit 34 Prozent gemessen an der Schadenhöhe die Hauptursache von Transportschäden. Seltenere Raubüberfälle führen insbesondere bei hochwertigen Sendungen zu relativ hohen Schadenaufwendungen.

Generell ist aus den Schadendaten zudem festzustellen, dass die Hauptgefahren von Transportschäden im Bereich des Warenumschlags sowie der Warenlagerung liegen. Während eines Warentransports in den unterschiedlichen Verkehrsträgern treten relativ gesehen weniger Schäden auf. Durch organisatorische Fehler und fehlende Harmonisierung bzw. Transparenz von Transportprozessen können zudem Lieferverzögerungen insbesondere bei verderblichen Waren wie Lebensmitteln oder Pharmazeutika schnell zu Qualitätsverlusten führen. Die in dieser Hinsicht möglichen Maßnahmen skizziert das folgende Kapitel. 


\subsection{Organisatorische Maßnahmen zur Schadenverhütung}

Die organisatorischen Schadenverhütungsmaßnahmen setzten an der Reduktion der Schadenwahrscheinlichkeit oder an der Reduzierung der Schadenhöhe an. Dabei nimmt die Auswahl der Transportmittel bzw. des Verkehrsträgers eine zentrale Rolle ein (siehe Tabelle 1). Transportmittel sollten auf die zu transportierenden Güter sowie die zu erwartenden Transportbedingungen (z. B. klimatisch) abgestimmt werden (Rogler 2002, S. 97). Zudem können neben den Zeit- und Kostenfaktoren auch die Risiko- bzw. Gefahrenaspekte einer Transportroute evaluiert und bei der Routenwahl berücksichtigt werden. Können schadenanfällige oder unsichere Routen dennoch nicht vermieden werden, werden Schutzmaßnahmen wie Begleitschutz oder zwei Fahrer zur Vermeidung von Ruhezeiten auf Parkplätzen empfohlen (Kummer/Sudy 2007, S. 265; Abele 2005, S. 6). Der folgende Abschnitt gliedert sich in Maßnahmen zur Risikodiversifikation, Verpackungsoptimierung sowie Anpassungen der Supply-ChainStrategie.

Die Verteilung der zu transportierenden Güter auf mehrere Teilsendungen begrenzt das Risiko hoher Schadenereignisse (Schulte 2009, S. 150). Bei großen Unternehmen ist dabei allein durch die hohen Sendungsvolumina eine Verteilung der Güter auf mehrere Teilsendungen unumgänglich. Doch speziell für kleinere und mittelgroße Unternehmen (KMU) ergibt sich hier eine Abwägung zwischen Kostenvorteilen durch die Bündelung von Sendungen und einer risikobehafteten Akkumulation der Güter z. B. in nur einem Seecontainer. Werden dann Waren beschädigt oder gehen verloren, kann dies bei den Kunden z. B. zu Betriebsunterbrechungen und für den Lieferanten zu ernsten wirtschaftlichen Konsequenzen (z. B. Nachproduktion, Auftragsverlust) führen.

Ein weiterer wichtiger Aspekt ist die Auswahl einer geeigneten Verpackung, die ihre Schutzfunktion möglichst optimal erfüllt. Auch hier muss zwischen kostenintensiven Spezialverpackungen, die auf das Transportgut genau zugeschnitten werden, und günstigen standardisierten Verpackungslösungen abgewogen werden. In der Praxis wird allerdings allzu oft die ausreichende Schutzfunktion zugunsten der Kosten vernachlässigt. Wildemann (1995) hat in diesem Zusammenhang den ungenügenden Schutz gegen Stöße, die falsche Einschätzung der klimatischen Bedingungen eines Transports und die ungenügende Kennzeichnung auf den Verpackungen als Probleme identifiziert. Außerdem können die Ansprüche hinsichtlich der Verpackungsgestaltung zwischen den Akteuren eines Transports unterschiedlich bzw. widersprüchlich sein (Vahrenkamp 2007, S. 328). Generell hat die Verpackung jedoch nicht nur Einfluss auf den Warenzustand, sondern ermöglicht auch einen rationelleren und umschlagseffizienten Transport beispielsweise durch die Verwendung standardisierter Lade- und Verpackungsmittel (Pfohl 2004, S. 147).

Neben diesen eher operativen Maßnahmen gibt es auch eine Reihe von Lösungsansätzen eher strategischer Natur. Häufig wird unter dem Begriff der lokalen Beschaffung und Distribution („local sourcing“/,/local distribution“) die geografische Annäherung 
der Transportakteure bezeichnet. Insbesondere in den eng getakteten Fertigungsprozessen der Automobilindustrie wird z. B. über Zulieferparks, welche nah am eigentlichen Werksgelände entstanden sind, das Transportrisiko durch eine Reduktion der Transportzeit auf ein Minimum beschränkt (Kummer/Sudy 2007, S. 264).

Durch eine verstärkte Zusammenarbeit im Beschaffungsbereich erhalten Unternehmen einen besseren Überblick über die zu transportierenden bzw. zu lagernden Güter. Im Rahmen dieser Kooperation werden alle Partner einer Transportkette integriert und es kann gemeinsam nach Optimierungspotenzialen zur Schadenverhütung gesucht werden. Mögliche Beispiele sind Supply-Chain-Konzepte wie „Efficient Customer Response“ (ECR), "Continuous Replenishment“ (CRP), "Quick Response“ (QR) oder "Collaborative Planning and Replenishment" (CPFR), die sich auf eine hohe Liefergeschwindigkeit und Kundennähe fokussieren (Seifert 2006; Suri 1998).

Speziell für den Bereich der straßengebundenen Transporte stellt auch eine gezielte Erhöhung der Verkehrssicherheit z. B. über Fahrertrainings im Nutzfahrzeugbereich ein wirksames Mittel dar. Gemäß einer aktuellen Studie der Axa Versicherung (2011) kommen auf 1.000 Flottenfahrer 90 bis 100 Unfälle pro Jahr, bei denen es sich häufig um schwerwiegende Unfälle mit Komplettverlusten handelt, sobald ein Lkw am Unfall beteiligt ist. Die Hauptursachen schwerer Unfälle im Transportgewerbe wie Unachtsamkeit, mangelnde Fahrtüchtigkeit (Übermüdung) und ungenügender Abstand könnten gezielt über wiederholende Trainings angegangen werden (Axa Versicherung 2011).

\subsection{Technologieorientierte Maßnahmen zur Schadenverhuitung}

Neben den organisatorischen Maßnahmen nehmen die technologieorientierten eine immer wichtiger werdende Rolle ein. Sie bieten nicht nur die Möglichkeit, Schäden zu verhindern oder deren Ausmaß zu reduzieren, sondern auch den Mehrwert in Form von Informationen über den Zustand der Güter, die dem Warenempfänger bereitgestellt werden können. Bei der Erfassung des Warenzustands kann analog zu den gängigen Verfahren im Bereich der Sendungsverfolgung zwischen diskreter und kontinuierlicher Erfassung unterschieden werden. Die diskrete Erfassung ermittelt die Transportkonditionen z. B. in zeitlich definierten Abständen oder bei Überschreitung von Toleranzwerten, die beide vor dem Transport an die zu erwartenden Transportkonditionen angepasst werden können. Bei der kontinuierlichen Erfassung werden die Konditionen über den gesamten Transport erfasst. Dabei werden deutlich mehr Daten erzeugt und der Informationsgehalt ist ungleich größer als bei den diskreten Verfahren. Allerdings benötigt eine kontinuierliche Datenerfassung größere Datenspeicher und der Energieverbrauch ist höher, was zu neuen Restriktionen bei Bau- und Batteriegröße führt. In jedem Fall lassen sich durch diesen Zugewinn an Informationen die Transportkonditionen und die schadenverursachenden Akteure von Transportprozes- 
sen identifizieren, auch wenn sich der Schadeneintritt nicht verhindern lässt. Zudem wird es möglich, die Transporte in Zukunft besser gegen die aufgetretenen Schäden bzw. Verluste zu schützen, da mehr Informationen über Art und Ursache der Schäden bekannt sind. Die verfügbaren Technologien zur Schadenverhütung lassen sich in die drei Kategorien Indikatoren, Daten-Logger und Telematik-Systeme einteilen.

Indikatoren ermöglichen eine kostengünstige Überprüfung der sachgerechten Behandlung eines Transportguts sowie die Identifikation von möglichen Beschädigungen während des Transports. Sie eignen sich daher für den flächendeckenden Einsatz bei schadenanfälligen Transportgütern. Indikatoren zeigen ein Überschreiten ihres definierten Toleranzbereiches meist durch farbliche, irreversible Kennzeichnung an. Allerdings prüfen Indikatoren oft nur einige wenige Schadenursachen. Eine allgemeine Erfassung der Transportkonditionen ist nur möglich, wenn für jede der identifizierten Risikoquellen (siehe Kapitel 2.2) ein Indikator auf dem Packstück platziert wird. Jedoch sind nicht alle Güter gegen alle fünf Risikoquellen gleichzeitig anfällig, so dass meist der Einsatz von ein bis zwei Indikatoren ausreicht, um die warenspezifischen Risikoquellen zu adressieren. Zusätzlich kann allein das Anbringen eines Indikators auf dem Packstück die Sensibilisierung des Logistikpersonals insbesondere beim Warenumschlag erhöhen. Zur Identifikation von Manipulationen der Waren oder dem Nachweis unbefugter Öffnungen von Packstücken dienen Sicherheitssiegel und Plomben, die ebenfalls unter die Gruppe der Indikatoren fallen. Teilweise kommen im Bereich der Indikatoren auch neue Lösungen aus dem Bereich der Radio-Frequenz-Identifikation (RFID) kombiniert mit einem Sensor zum Einsatz.

Daten-Logger zeichnen Transportkonditionen kontinuierlich bzw. zeit-diskret auf und speichern neben den Messwerten auch den Zeitpunkt der Messung auf. Durch Integration verschiedener Sensoren (z. B. Temperatur, Feuchte, Beschleunigung, Neigung etc.) können Daten-Logger auch mehrere Risikoquellen gleichzeitig überwachen. Anders als bei Indikatoren können die Daten-Logger den Zeitpunkt eines Schadens genau bestimmen. Mit dieser Datenspeicherung können die Transportrouten hinsichtlich ihrer Risikoquellen langfristig optimiert werden. Dazu müssen die Daten-Logger allerdings meist mit Hilfe spezieller Software ausgelesen werden, was den Administrationsaufwand erhöht. Die Analyse kann dabei vom Empfänger der Ware oder nach Rückführung des Daten-Loggers vom Versender durchgeführt werden. Typische Einsatzgebiete von Daten-Loggern sind Branchen mit hochwertigen und teils sehr empfindlichen Gütern wie Pharmazeutika, Medizintechnik oder aus dem Bereich des Maschinen- und Anlagenbaus sowie der Elektronik. Je nach Häufigkeit der Messungen können Daten-Logger auch über mehrere Monate im Einsatz sein.

Telematik-Systeme verfügen wie die Daten-Logger über verschiedene Sensoren, zusätzlich jedoch über ein Kommunikations- und Ortungsmodul. So ist es möglich, die Transportkonditionen nicht nur aufzuzeichnen, sondern auch die erfassten Daten direkt an die Akteure des Transportprozesses weiterzuleiten. Voraussetzung ist jedoch eine ausreichende Netzabdeckung. Zur Übertragung der Daten eignen sich prinzipiell die Mobilfunk- oder Satellitenkommunikation, in Nahbereichen z. B. innerhalb von 
Häfen über Wireless LAN-(WLAN-)Netzwerke möglich. Ist die Datenkommunikation nicht möglich, speichern die Telematik-Systeme die aufgezeichneten Daten lokal zwischen. Durch diese aktive Benachrichtigung bei kritischen Transportkonditionen können die Akteure anbahnende Schäden frühzeitig identifizieren und - sofern möglich Maßnahmen zur Schadenreduktion ergreifen. Besonders bei Schäden durch Temperatur- und Nässeeinwirkung besteht eine gewisse Toleranzzeit, die aktiv zur Schadenabwehr genutzt werden kann. Auch bei Diebstahl und Verlust ist es durch die Lokalisierung der Güter möglich, diese schnell wiederzufinden oder ein Warendiebstahl z. B. durch Routenabweichungen früh zu identifizieren. In diesem Fall können ebenso Maßnahmen zur Schadenreduktion ergriffen werden. Zusätzlich erleichtert der Informationsaustausch die Optimierung der Transportabläufe.

\section{$3 \quad$ Maßnahmenraster zur Schadenverhuitung}

\subsection{Methodik zur Bewertung des Technologieportfolios}

Auf der Basis der Grundlagen in Kapitel 2 wird im Kapitel 3 das technologieorientierte Maßnahmenraster zur Schadenverhütung von Transportschäden entwickelt. Um für Praktiker möglichst hilfreiche Handlungsempfehlungen ableiten zu können, wird bei der Bewertung von Technologien auf Portfoliomethoden zurückgegriffen. Sie erlauben eine Systematisierung einer ersten Wirtschaftlichkeitsbewertung auf der Grundlage von mehrstufigen qualitativen Bewertungskriterien (Haag/Schuh/Kreysa/Schmelter 2011, S. 330). Unternehmen, die eine Investition in technologieorientierte Schadenverhütungsmaßnahmen evaluieren, besitzen mit dem folgenden Raster eine mögliche Grundlage für Investitionsentscheidungen.

Generell stehen Unternehmen vor der Frage, inwiefern die technologieorientierten Schadenverhütungsmaßnahmen zu den eigenen wettbewerbsrelevanten Kompetenzen des Unternehmens passen. Insbesondere besteht durch den Technologieeinsatz auch die Möglichkeit, sich über Schadenverhütung bzw. Qualitätsverpflichtungen aktiv am Markt zu differenzieren.

Die diesem Beitrag zugrunde liegende Methodik zur Technologiebewertung orientiert sich an der von Pfeiffer (1991) entwickelten Technologieportfolioanalyse (TPF-Analyse). Dieser Ansatz ordnet die in Frage kommenden Technologien nach ihrer Attraktivität und Ressourcenstärke und schätzt deren zukünftige Entwicklung ab (Haag et al. 2011, S. 330). Der Ansatz favorisiert dabei die frühzeitige Investition in relevante Technologien, um damit eine Pionierstrategie zu verfolgen. Somit lässt sich dieses Vorgehen mit dem Modell von Strebel (2007) vergleichen, der die Technologien nach deren Gegenstandsbereich, dem Verbreitungs- und Neuheitsgrad sowie dem Funktionsumfang einteilt. Die TPF-Analyse folgt im Wesentlichen einem vierstufigen Pro- 
zess, in den zur Bewertung der technologieorientierten Schadenverhütungsmaßnahmen auch Experten eingebunden wurden (Pfeiffer/Metze/Schneider/Amler 1982, S. 78):

In der ersten Stufe werden alle von einem Unternehmen zur Schadenverhütung eingesetzten Technologien und Maßnahmen identifiziert.

In der zweiten Stufe wird die Bewertung der Technologien anhand einer Matrix hinsichtlich deren technischer Bedeutung und dem Vorsprung bzw. Rückstand des Unternehmens in diesen Technologien abgebildet.

Es folgt in der dritten Stufe die Transformation des Portfolios in einen zukünftigen Zeitpunkt. Dabei werden alle zukunftsträchtigen Technologien des Unternehmens sowie die notwendigen Ressourcen zur Aufholung des identifizierten Rückstands erfasst.

Zum Abschluss werden in der letzten, vierten Stufe konkrete Handlungsempfehlungen entwickelt, die in diesem Beitrag als eigenständiges Kapitel 4 angelegt sind.

Da die TPF-Analyse in ihrer ursprünglichen Form sehr unternehmensspezifisch ist, das entwickelte Raster in diesem Beitrag allerdings möglichst allgemein gehalten werden soll, wurde die Matrix angepasst. Diese zeigt nun auf der Abszisse nicht mehr die effektive Ressourcenstärke des Unternehmens auf, sondern die notwendige Ressourcenstärke, die ein Unternehmen zum Einsatz der technologieorientierten Schadenverhütungsmaßnahmen benötigt. Die Ressourcenstärke setzt sich aus den Dimensionen Finanzen (Kosten) und Wissensintensität zusammen (Pfeiffer et al. 1982, S. 89). Die Ordinate gibt unternehmensunabhängig die Attraktivität der Technologie zur Schadenverhütung an, die auf dem Nutzenpotenzial der Technologie, der Anwendungsbreite und der Kompatibilität als Dimensionen basiert.

\subsection{Entwicklung des Maßnahmenrasters}

Die Ausführungen der Kapitel 3.2 und 3.3 basieren auf einer leitfadengestützen Expertenbefragung, die zwischen Ende April und Anfang Mai 2011 mit insgesamt vier Experten durchgeführt wurde. Die Zielsetzung der Expertenbefragung war, den eigenen Modellentwurf der zuvor aus der Theorie abgeleiteten Technologieportfolios zu justieren. Damit wird die Validität des in diesem Beitrag entwickelten Maßnahmenrasters sichergestellt. Die Experten wurden zu Chancen, Nutzenpotenzialen sowie möglichen Risiken bei der Einführung und langfristigen Umsetzung von technologieorientierten Schadenverhütungsmaßnahmen befragt.

Alle geführten Interviews wurden aufgezeichnet, um die anschließende Transkription der Interviews zu erleichtern (Bortz/Döring 2006). Die wörtliche Transkription wurde allerdings vor der Auswertung in Schriftdeutsch übertragen (Mayring 2008). Bei der Auswertung der transkribierten Experteninterviews wurden die Aussagen zu Katego- 
rien zusammengefasst. Diese Form der Inhaltsanalyse bietet bei der Auswertung eine systematische und regelgeleitete Vorgehensweise (Gläser/Laudel 2009; Mayring 2008).

Die Tabelle 2 zeigt eine Übersicht über die Interviewpartner aus der Industrie.

Tabelle 2: $\quad$ Übersicht über die befragten Experten

\begin{tabular}{|l|l|l|}
\hline Name/ Position & Unternehmen & Datum \\
\hline $\begin{array}{l}\text { Beat Glauner, Geschäftsführer und } \\
\text { Inhaber }\end{array}$ & VTT AG, Muttenz (CH) & 04.05 .2011 \\
\hline $\begin{array}{l}\text { Andreas Kindler, Key-Account } \\
\text { Manager und Projektleiter }\end{array}$ & Egolf Verpackungs AG, Zürich-Altstetten (CH) & 28.04 .2011 \\
\hline $\begin{array}{l}\text { Christopher Regenhardt, Produkt- } \\
\text { manager }\end{array}$ & Schenker AG, Essen (DE) & 04.05 .2011 \\
\hline Niels Wildner, Verkaufsleiter & TCl GmbH, Oststeinbek (DE) & 19.04 .2011 \\
\hline
\end{tabular}

Im folgenden Abschnitt werden die Technologieportfolios für jeden zuvor identifizierten Risikofaktor (vgl. Kapitel 2.2) separat entwickelt und die drei Technologiekategorien Indikatoren, Daten-Logger und Telematik-Systeme darin eingeordnet. Die Einteilung wurde gemeinsam mit den Experten festgelegt, die durchweg Lieferanten der drei Technologiekategorien sind.

\subsubsection{Erschütterung}

Zur Schadenverhütung bzw. zur Identifikation von Schäden aus dem Bereich Erschütterung eignen sich Stoßindikatoren, „Shock Recorder“ aus der Gruppe der DatenLogger und das Telematik-System mit einem Beschleunigungssensor. In der Praxis verbreitet eingesetzt werden vor allem die Stoßindikatoren (z. B. Shockwatch), um die möglichen Beschädigungen möglichst frühzeitig auf dem Transport zu erkennen. Hauptanwendungsgebiet sind bisher erschütterungsempfindliche Güter wie Maschinen und elektronische Bauteile. Großes Potenzial birgt auch das Telematik-System, das mit einer zunehmenden Kostendegression generell in Zukunft noch an Bedeutung gewinnen wird. Vor allem die aktive Information über Beschädigungen während des Transports erhöht die Transparenz auf Transportkonditionen. Nach dem Eintritt des Schadenereignisses kann aufgrund der Datenlage entschieden werden, ob z. B. eine Maschine überhaupt weitertransportiert wird, vor Ort untersucht oder zurück an den Hersteller zur Überholung bzw. Reparatur gesendet werden sollte. Da Erschütterungen meist abrupt auftreten, ist es für Schadenverhütungsmaßnahmen im Schadeneintritt eigentlich zu spät. Die konsequente Datenerfassung und -auswertung ermöglicht jedoch die langfristige Optimierung z. B. von Transportrouten oder auch, konstruktionsbedingte Schwachstellen für Erschütterungen zu beheben. 
Abbildung 3: Technologieportfolio, Maßnahmen gegen Risikoquelle Erschütterung

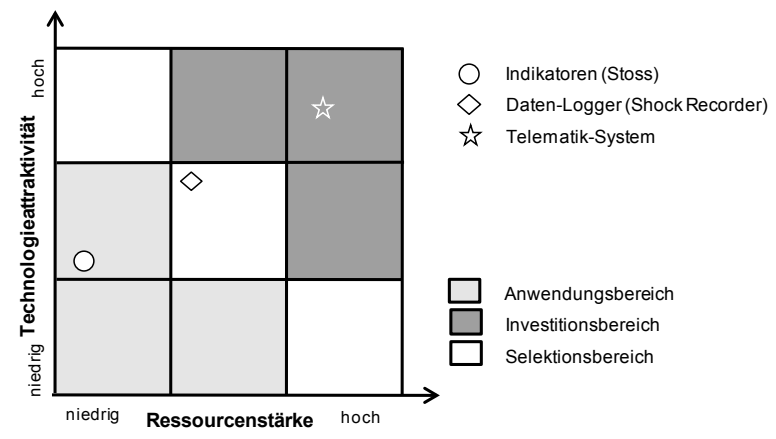

Abbildung 3 zeigt die Technologieportfolio-Matrix für die Risikoquelle Erschütterung. Indikatoren sind im Anwendungsbereich positioniert, während das Telematik-System im Investitionsbereich eingeordnet ist. Das bedeutet, Indikatoren können über Lieferanten günstig bezogen werden, Telematik-Systeme jedoch sind vergleichsweise teuer und müssen teilweise vom Transportkunden noch an die Transportgegebenheiten angepasst werden. Dafür tragen Telematik-Systeme zur Differenzierung des Transportkunden am Markt insbesondere als Qualitätsführer bei, der auch die Transportkette bestmöglich überwacht und die Qualität bis zum Endkunden. Daten-Logger sind im Selektionsbereich abgebildet, in dem ein situatives Vorgehen notwendig ist.

\subsubsection{Diebstahl und Verlust}

Für die Schadenverhütung von Diebstahl und Verlust eignen sich nur zwei der drei Technologien. Indikatoren im Sinne der weit verbreiteten Siegel und Plomben sind günstig und benötigen zur Anwendung kaum spezielles Wissen. Siegel werden z. B. an allen Standardcontainern in der Seefracht angebracht, um den zwischenzeitlichen Zugriff z. B. im Rahmen der Verzollung zu kontrollieren. In der Luftfracht und im inländischen Landtransport sind Siegel und Plomben jedoch nur selten im Einsatz. Trotzdem können durch die Verwendung dieser Indikatoren unbefugte Zugriffe auf die Transportgüter identifiziert werden. Am anderen Ende des Technologieportfolios sind Telematik-Systeme positioniert, die die genaue Position regelmäßig bestimmen sowie z. B. über Türüberwachung und Bewegungsmelder im Innenraum verfügen. Bei Abweichungen von zuvor definierten Soll-Werten oder Korridoren alarmiert das System selbstständig einen zuvor definierten Personenkreis. Derart ausgerüstet können Diebstahl und Raub sehr früh erkannt und mögliche Gegenmaßnahmen zur Schadenverhütung ergriffen werden. Zudem wird die Wiederauffindbarkeit verlorener Waren erleichtert. Die Abbildung 4 stellt das Technologieportfolio entsprechend dar. 
Abbildung 4: Technologieportfolio, Maßnahmen gegen Risikoquellen Diebstahl/Verlust

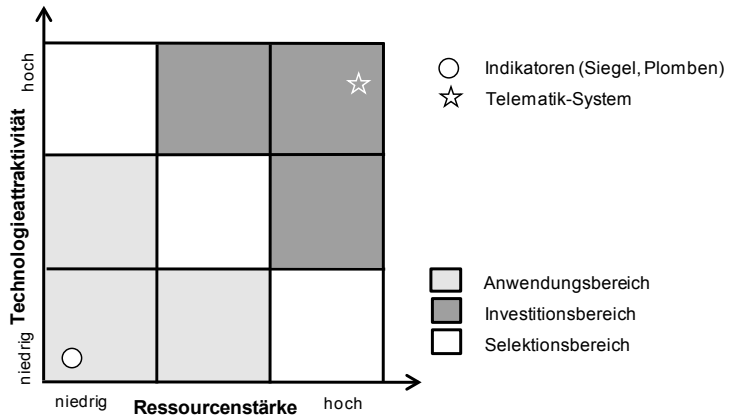

\subsubsection{Kippen und Neigung}

Schadenverhütungsmaßnahmen für die Risikoquellen Neigung und Kippen lassen sich in allen drei Technologiebereichen finden. Speziell die Neigungsindikatoren sind technisch nicht sehr anspruchsvoll und zeigen dennoch ein Kippen der Waren zuverlässig an. Die Stückkosten für Indikatoren liegen auch im Vergleich zu anderen Indikatoren am unteren Rand. Neigungs-Logger bieten den Vorteil konkreter Messwerte, die, kontinuierlich erfasst, mehr Rückschlüsse auf den Kippvorgang und die Zeitperiode, in der das Packstück gekippt transportiert wurde, zulassen. Eine Analyse der Daten ist allerdings erst im Nachhinein möglich.

Abbildung 5: Technologieportfolio, Maßnahmen gegen Risikoquellen Neigung/Kippen

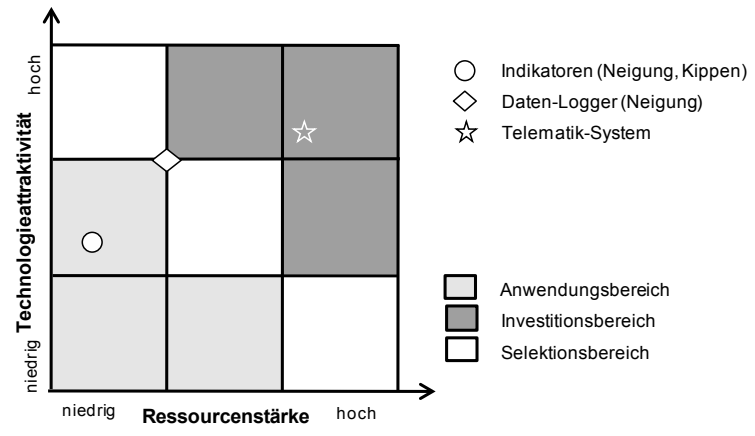

Telematik-Systeme sind für diese Risikoquelle wenig verbreitet, obwohl sich Messwerte bei Neigung und Kippen von Gütern generell übertragen lassen. Insgesamt spielt 
die Transportüberwachung im Bereich Neigung eher eine Nebenrolle und wird meist mit Indikatoren durchgeführt oder gemeinsam mit der Überwachung von Erschütterungen eingesetzt.

\subsubsection{Temperatureinfluss}

Maßnahmen zur Temperaturüberwachung und die Identifikation von Abweichungen haben in den vergangenen Jahren stark an Bedeutung gewonnen. Insbesondere in der Lebensmittel-, Pharma- und in Teilen der Chemiebranche sind temperaturüberwachte Transporte z. B. aus Produkthaftungsgründen vorgeschrieben. Außerdem versuchen Speditionen durch Speziallösungen, sich im Bereich der temperaturgeführten Transporte vom Wettbewerb zu differenzieren. Neben diversen Temperaturindikatoren, die je nach Kalibrierung unterschiedliche Temperaturkorridore überwachen, sind vor allem Daten-Logger sehr verbreitet. Etwas komplexer ist die Programmierung der Temperaturzonen bei den Daten-Loggern, die für die jeweiligen Transportbedingungen vor dem eigentlichen Transport manuell konfiguriert werden müssen. Dabei können vielfach bis zu acht verschiedene Temperaturgrenzen definiert werden. TelematikSysteme, die allein zur Temperaturüberwachung eingesetzt werden, sind verhältnismäßig teuer und werden nur bei sehr sensiblen Gütern wie z. B. Impfstoffen und Testsubstanzen in der Praxis gebraucht. Für diese hochwertigen Warengruppen ist der Telematik-Einsatz dann auch aus Diebstahl/Verlust-Perspektive sinnvoll. Abbildung 6 stellt das Technologieportfolio für die Risikoquelle Temperatureinfluss dar.

Abbildung 6: Technologieportfolio, Maßnahmen gegen Risikoquelle Temperatur

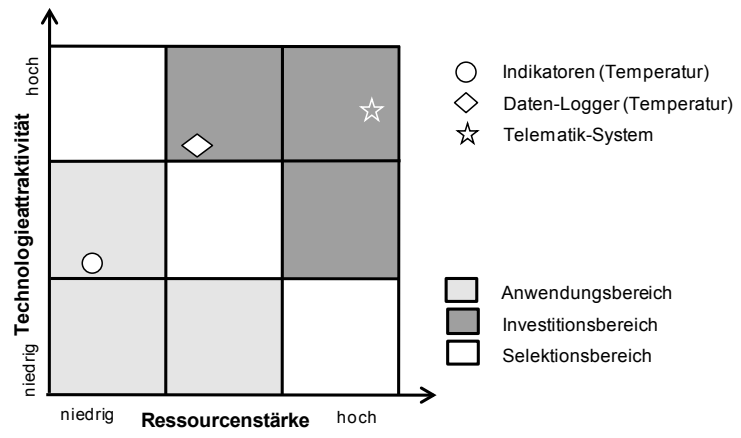

\subsubsection{Einwirkung von Nässe und Feuchtigkeit}

Typische Anwendungsfelder für eine Transportüberwachung in Bezug auf die Einwirkung von Nässe und Feuchtigkeit finden sich im Bereich der Seefracht, um dort insbesondere die Gefahr der Korrosion und Schimmelbildung bei den Waren zu identifizie- 
ren. Doch auch im Landverkehr ist der Einsatz von Feuchtigkeitsindikatoren möglich, da z. B. bei längeren Tunnelstrecken die Luftfeuchte im Laderaum gefährlich ansteigen kann. Bei der Überwachung der Luftfeuchtigkeit während des Transports messen viele Geräte neben der eigentlichen Feuchtigkeit zusätzlich auch die Umgebungstemperatur. Damit kann kontinuierlich der Taupunkt bestimmt werden, bei dessen Überschreitung die Feuchtigkeit aus der Luft kondensiert und sich als Wassertropfen auf den Transportgütern absetzt. Indikatoren basieren auf chemischem Indikatorpapier, das sich bei unterschiedlichen Feuchtigkeitsniveaus verfärbt. Daten-Logger und Telematik-Systeme verfügen über Feuchtigkeits- und Temperatursensoren und können beide z. B. durch akustische Signale bei Überschreitung des Taupunkts Logistikmitarbeiter warnen. Letztgenanntes Szenario ist auch bei der Zwischenlagerung der Transportgüter während des Transports zur Vermeidung von Korrosion und Schimmelbildung in der Praxis häufiger anzutreffen.

Abbildung 7: Technologieportfolio, Maßnahmen gegen Risikoquelle Nässe bzw. Feuchte

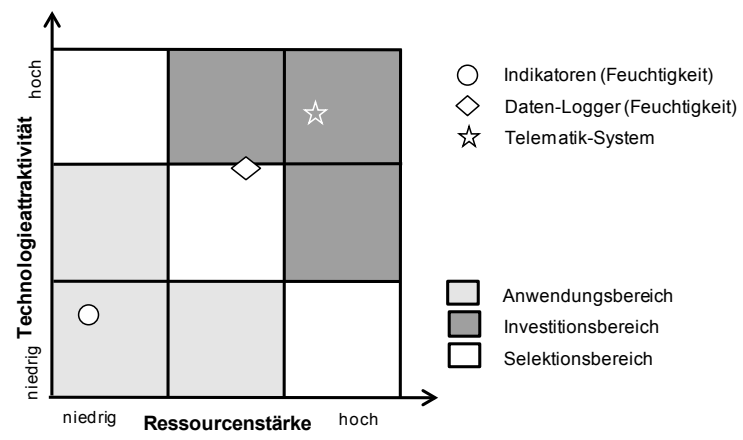

\subsection{Expertenbefragung zu Schadenverhütungspoten- zialen technologieorientierter Maßnahmen}

Generell weisen die Experten darauf hin, dass Schadenverhütung nicht alleine auf die drei Technologiekategorien Indikatoren, Daten-Logger und Telematik-Systeme reduziert werden darf. Entscheidend bei der Identifikation der jeweiligen unternehmensspezifischen Maßnahmen ist, die Lieferkette und das bestehende Schadensbild des Unternehmens vorab genau zu analysieren. Die Bandbreite der Beratungen ist dabei sehr breit und reicht z. B. vom Vorschlag einer alternativen Transportroute über Verpackungsoptimierung bis hin zur Anpassung der administrativen Vorgänge.

Welche konkreten Schadenverhütungsmaßnahmen in einem Unternehmen umgesetzt werden, hängt von zahlreichen Faktoren ab. Die Experten identifizieren insbesondere die Branche des Versenders, den Warenwert und die Anfälligkeit des Gutes als ent- 
scheidende Faktoren. Daneben wirken hohe Konventionalstrafen bei Lieferschwierigkeiten sowie auch aktuelle "Green Logistics“-Projekte förderlich auf die Einführung der Transportüberwachung bzw. auf die Erhöhung der Transparenz in der Transportkette. Durch eine verbesserte Koordination der Prozesse können die zeitlichen Abläufe optimiert, die Auslastung der Transportmittel gesteigert und die Zuverlässigkeit der Transportprozesse verbessert werden.

Durch die laufende Überwachung der Transportkonditionen können zudem die Verpackungen immer wieder angepasst werden, so dass die Waren optimal vor Beschädigungen geschützt werden. So besteht auch die Gefahr einer unnötigen Überverpackung der Güter nicht. Folglich könnten die Verpackungskosten unter Beibehaltung der Schutzfunktion reduziert werden. Weitere Aspekte sind der Aufbau von internem Wissen und eine Sensibilisierung für die Risiken und Gefahren der unternehmensspezifischen Transportprozesse.

Insgesamt können sich Investitionen in Schadenverhütung über eine steigende Qualität der Waren durchaus für die Unternehmen auszahlen. Selbst bei Schäden infolge Erschütterung oder Kippen der Waren können durch die frühzeitige Identifikation des Schadens negative Folgen über mögliche Alternativen (z. B. eine Ersatzlieferung per Express) verhindert oder abgemildert werden. Um die Investitionen vor allem in teure Daten-Logger und Telematik-Systeme zu begrenzen, bieten die Lieferanten meist Mietmodelle an, die in der Regel auch die Datenanalyse und Wartung beinhalten.

Durch die Einführung und konsequente Umsetzung von Schadenverhütungsmaßnahmen ergeben sich weitere eng verknüpfte Vorteile. Können die Schäden effektiv reduziert werden, sinken Schadenkosten und somit auch die Versicherungskosten bei versicherten Transporten. Die erhöhte Transparenz und die frühzeitige Identifikation von Beschädigungen und Verlusten können die Qualitätsprüfung beim Warenempfänger positiv beeinflussen. Insbesondere reduziert sich gemäß den Experten die Kontrollzeit, so dass die Güter früher der Weiterverarbeitung bzw. dem Verkauf zur Verfügung stehen.

Als Risiken bei der Umsetzung von technologieorientierten Schadenverhütungsmaßnahmen identifizieren die Experten eine mangelnde Sensibilisierung der Versender, da Schadenverhütungsmaßnahmen bisher fast ausschließlich nur auf Kundendruck oder Empfehlung ihrer Versicherung eingeführt würden. Fehlt die notwendige Auseinandersetzung mit der Thematik, verlieren die Maßnahmen ihre Wirkung. Außerdem versuchen Finanz- und Controllingabteilungen aktiv, Schadenverhütungsmaßnahmen aus Kostengründen zurückzufahren, bis die Transportqualität auf ein nicht mehr wettbewerbsfähiges Niveau sinkt. Erst dann würden Unternehmen wieder im Rahmen von neuen Qualitätsprogrammen in Schadenverhütungsmaßnahmen investieren. Dieser Zyklus, so berichten die Experten, wiederholt sich etwa alle fünf bis sieben Jahre.

Zudem weisen die Experten darauf hin, dass speziell ein ausgelöster Indikator zu Verunsicherungen in der Transportkette führen könnte. Spediteure und Frachtführer nehmen dann in der Regel die Sendung nicht mehr an bzw. transportieren diese ohne 
Zustimmung des Auftraggebers oder Funktionsprüfung weiter. Generell entstehen durch den Einsatz von Schadenverhütungsmaßnahmen neue manuelle und administrative Prozesse, die ebenfalls ein Risiko in sich tragen. Die am Transport beteiligten Mitarbeiter müssen geschult, die Geräte fachmännisch an den Packstücken angebracht, kalibriert und entfernt werden. Somit gehen die Vorteile einer Schadenverhütung mit neuen Risiken einher, die ihrerseits bewertet werden müssen. Werden Risiken vernachlässigt, besteht die Gefahr, dass einerseits das Wirkungspotenzial der Maßnahmen verloren gehen und andererseits neue Schäden entstehen könnten, die ohne den Einsatz der Maßnahmen nicht aufgetreten wären.

\section{$4 \quad$ Handlungsempfehlungen für die Praxis}

Nach der Aufarbeitung der Schadenverhütungsthematik, Entwicklung des Maßnahmenrasters und der Expertenbefragung aus der Transportüberwachungs- wie auch Verpackungsbranche werden in diesem Kapitel die abgeleiteten Handlungsempfehlungen für die Praxis zusammengestellt.

Grundvoraussetzung dafür, dass die von einem Unternehmen getroffenen Schadenverhütungsmaßnahmen greifen, ist die Sensibilisierung der am Transport beteiligten Mitarbeiter intern sowie extern. Die Sensibilisierung legt somit das Fundament für die weitere Umsetzung und den Erfolg der getroffenen Maßnahmen. Die in diesem Beitrag vorgestellten Technologiekategorien unterstützen dies im Allgemeinen. Zur Bewertung und Auswahl der jeweils geeigneten Maßnahmen sollten Unternehmen auf ihre vorhandene Schadenhistorie und ihre Transportcharakteristiken zurückgreifen. Denn erst wenn die Schwachstellen einer Transportkette bekannt sind, können diese zielgerichtet adressiert werden. Darüber hinaus benötigen die Technologielieferanten möglichst genaue Anforderungen, damit ihre eigene Beratung möglichst nah an den Schwachstellen des Unternehmens im Transport ansetzt.

Nach einer erfolgreichen Implementierung und Umsetzung der Schadenverhütungsmaßnahmen sollten Abweichungen oder Änderungen an den Transportsendungen kommuniziert werden. Nur so bleibt es gewährleistet, dass Indikatoren, Daten-Logger und Telematik-Systeme korrekt konfiguriert sind und auch nur wirkliche Schadenereignisse anzeigen. Gerade in der mangelnden Kommunikation liegt eines der Hauptrisiken bei der Umsetzung von Maßnahmen. Hier hilft vor allem ein ernsthaftes Engagement der Unternehmensführung.

Richtig umgesetzt tragen die Schadenverhütungsmaßnahmen jedoch dazu bei, Kosten im Bereich der Schäden, Verpackung und Versicherung zu reduzieren. Die Erhöhung der Transparenz auf die Transportprozesse führt zu einem langfristigen Aufbau von Wissen und Erfahrung innerhalb des Unternehmens. Die Sensibilisierung der Mitarbeiter auf Transportschäden steigert die Sorgfalt und Umsichtigkeit im Warenumschlag. So nimmt die Reputation eines Unternehmens durch Qualitätsverbesserungen 
infolge der Maßnahmen langfristig $\mathrm{zu}$, und heute noch weit verbreitete negative Kettenreaktionen entlang der Transportkette wie Lieferausfälle, Qualitätsschwankungen, Konventionalstrafen und der Verlust von Kunden können verringert werden. Die in diesem Kapitel vorgestellten Technologieportfolios können Unternehmen bei der Auswahl der geeigneten Maßnahmen wirkungsvoll unterstützen.

\section{$5 \quad$ Zusammenfassung und Ausblick}

Das in diesem Beitrag vorgestellte technologieorientierte Maßnahmenraster zur Schadenverhütung und die anschließende Expertenbefragung rücken die Sensibilisierung der Unternehmen und Mitarbeiter einer Transportkette für die Thematik als zentrale Aussage ins Zentrum. Die in der Literatur zu findenden Lösungsansätze konzentrieren sich zu sehr auf strategische Maßnahmen wie eine Reorganisation der Beschaffung und des Vertriebs. In seltenen Fällen jedoch werden erste operativ hilfreiche Maßnahmen zur Unterstützung der Schadenprävention erwähnt. Der in diesem Beitrag vorgestellte Ansatz setzt hier unmittelbar an. Die sinnvolle Kombination aus Transportsicherung, Verpackungsoptimierung und Transportüberwachung eröffnet eine Vielzahl an Optimierungsmöglichkeiten zur Verbesserung der Transportqualität und der Schadenprofile im Unternehmen.

Sicher jedoch ist, dass die Bereitschaft, sich mit dem Thema Transportsicherheit und Schadenverhütung auseinanderzusetzen, in Zukunft weiter zunehmen wird. Entweder aus eigener Überzeugung der Unternehmen bzw. ihrer Mitarbeiter oder aufgrund von externem Druck.

\section{Literatur}

Abele, E. (2005): Versicherung und Schäden. In: Abele, E.; Teich, D.I. (Hrsg.): Praxishandbuch Transport, Weka Media: Kissing, S. 1-30.

Autry, C.W.; Bobbit, L.M. (2008): Supply chain security orientation: conceptual development and a proposed framework. In: International Journal of Logistics Management, 19(1), S. 42-64.

Axa Versicherung (2011): Driving at work - Das Sicherheitspaket der Axa.

Bortz, J.; Döring, N. (2006): Forschungsmethoden und Evaluation für Human- und Sozialwissenschaftler, 2. überarb. Aufl., Springer: Heidelberg.

Christopher, M.; Peck, H. (2004): Building the resilient supply chain, International Journal of Logistics Management, 15(2), S. 1-13. 
Christopher, M; Towill, D. (2002): Developing Market Specific Supply Chain Strategies. In: International Journal of Logistics Management, 15(2), S. 1-14.

Eberle, A.-O. (2005): Risikomanagement in der Beschaffungslogistik - Gestaltungsempfehlungen für ein System, Dissertation 3008, Bamberg.

Gläser, J.; Laudel, G. (2009): Experteninterviews und qualitative Inhaltsanalyse, 3. Aufl., Gabler: Wiesbaden.

Haag, C.; Schuh, G.; Kreysa, J.; Schmelter, K. (2011): Technologiebewertung. In: Schuh, G.; Klappert, S. (Hrsg.): Technologiemanagement - Handbuch Produktion und Management 2, Springer: Berlin, S. 309-366.

Jüttner, U. (2005): Supply chain risk management - Understanding the business requirements from a practitioner perspective, International Journal of Logistics Management, 16(1), S. 120-141.

Jüttner, U.; Peck, H.; Christopher, M. (2003): Supply chain risk management: outlining an agenda for future research. In: International Journal of Logistics Research and Applications, 6(4), S. 197-210.

Kajüter, P. (2007) Risikomanagement in der Supply Chain: Ökonomische, regulatorische und konzeptionelle Grundlagen. In: Vahrenkamp, R.; Siepermann, C. (Hrsg.): Risikomanagement in Supply Chains, E. Schmidt: Berlin, S. 13-28.

Kersten, W.; Blecker, T. (2006): Managing Risks in Supply Chain - How to build reliable collaboration in logistics, E. Schmidt: Berlin.

Knemeyer, M. A.; Zinn, W.; Eroglu, C. (2009): Proactive planning for catastrophic events in supply chains, Journal of Operations Management, 27(4), S. 141-153.

Kummer, S; Sudy, I. (2007): Management von Transport- und Lagerrisiken in Supply Chains. In: Vahrenkamp, R.; Siepermann, C. (Hrsg.): Risikomanagement in Supply Chains, E. Schmidt: Berlin, S. 255-272.

March, J. G.; Shapira, Z. (1987): Managerial perspectives on risk and risk taking. In: Management Science, 33(11), S. 1404-1418.

Mason-Jones, R; Towill, D. (1998): Shrinking the supply chain uncertainty circle, In: IOM Control Magazine, 24(7), S. 17-22.

Mayring, P. (2008): Qualitative Inhaltsanalyse. Grundlagen und Technik, 10. Aufl., Beltz: Weinheim.

Meuser M.; Nagel, U. (2005): ExpertInneninterviews - vielfach erprobt, wenig bedacht. In: Bogner, A.; Littig, B.; Menz, W. (Hrsg.): Das Experteninterview, 2. Aufl., VS Verlag, S. 71-94. 
Norrman, A.; Jansson, U. (2004): Ericsson's proactive supply chain risk management approach after a serious sub-supplier accident In: International Journal of Physical Distribution \& Logistics Management, 34(5), S. 434-456.

Peck, H. (2005): Drivers of supply chain vulnerability: an integrated framework. In: International Journal of Physical Distribution \& Logistics Management, 35(4), S. 210-232.

Pfeiffer, W. (1991): Technologie-Portfolio zum Management strategischer Zukunftsgeschäftsfelder, 6. Aufl., Vandenhoeck und Ruprecht: Göttingen.

Pfeiffer, W.; Metze, G. Schneider, W.; Amler, R. (1982): Technologie-Portfolio zum Management strategischer Zukunftsgeschäftsfelder. Vandenhoeck und Ruprecht: Göttingen.

Pfohl, H.-C.; Köhler, H.; Thomas, D. (2010): State of the art in supply chain risk management re-search: empirical and conceptual findings and a roadmap for the implementation in practice, In: Logistics Research, 2, S. 33-44.

Pfohl, H.-C. (2004): Logistiksysteme, 7. Aufl. Springer: Heidelberg.

Pfohl, H.-C. (2002): Risiken und Chancen: Strategische Analyse in der Supply Chain. In: Pfohl, H.-C. (Hrsg): Risiko- und Chancenmanagement in der Supply Chain, E. Schmidt: Berlin, S. 1-56.

Rogler, S. (2002): Risikomanagement im Industriebetrieb: Analyse von Beschaffungs-, Produktions- und Absatzrisiken, DUV: Wiesbaden.

Schulte, C. (2009): Logistik - Wege zur Optimierung der Supply Chain, Vahlen: München.

Seifert, D. (2006): Efficient Consumer Response: Supply Chain Management (SCM), Category Management (CM) and Collaborative Planning, Forecasting and Replenishment (CPFR) als neue Strategieansätze, 4. Aufl., Mering.

Skorna, A.; Hinz, A. (2011): Warentransport ist risikoreich. In: io management, April 2011, S. 22-27.

Skorna, A.; Bode, C.; Fleisch, E. (2010): Technologiebasiertes Risikomanagement in globalen Transportnetzwerken. In: Industrie Management, 26(5), S. 14-18.

Skorna, A.; Bode, C.; Wagner, S. M. (2009): Technology-Enabled Risk Management along the Transport Logistics Chain. In: Wagner, S.M.; Bode, C. (Hrsg.): Managing Risk and Security, Haupt: Bern, S. 197-220.

Steven, M.; Pollmeier, I. (2007): Management von Kooperationen in Supply Chains. In: Vahrenkamp, R.; Siepermann, C. (Hrsg.): Risikomanagement in Supply Chains, E. Schmidt: Berlin, S. 273-286.

Strebel, H. (2007): Innovations- und Technologiemanagement, 2. Aufl., Facultas: Wien. 
Suri, R. (1998): Quick Response Manufacturing - A Company Wide Approach to reducing Lead Time, Productivity Press: Portland, OR.

Vahrenkamp, R. (2007): Logistik, Oldenbourg: München.

Wagner, F. (2010): Gabler Versicherungslexikon, Gabler: Wiesbaden.

Wagner, S. M.; Bode, C. (2007): An empirical investigation into supply chain vulnerability. In: Journal of Purchasing \& Supply Management, 12, S. 301-312.

Wildemann, H. (1995): Behältersysteme - Konzepte zur Optimierung des Behälterkreislaufs, TCW: München.

Zsidisin, G. A.; Ellram, L. (2003): An agency theory investigation of supply chain risk management. In: Journal of Supply Chain Management, 39(3), S. 15-27.

Zsidisin, G.A. (2001): Measuring supply risk: an example from Europe. In: Best Practices in Purchasing and Supply Chain Management, CAPS research, 4(3), S. 1-6. 